\title{
Parallel and selective trapping in a patterned plasmonic landscape
}

\author{
MAURIZIO RIGHINI ${ }^{1}$, ANNA S. ZELENINA ${ }^{1}$, CHRISTIAN GIRARD ${ }^{2}$ AND ROMAIN QUIDANTT1,3* \\ ${ }^{1}$ ICFO-Institut de Ciencies Fotoniques, Mediterranean Technology Park, 08860 Castelldefels (Barcelona), Spain \\ ${ }^{2}$ CEMES, UPR-CNRS 8011, 29 rue Jeanne Marvig, 31055 Toulouse, France \\ ${ }^{3}$ ICREA-Institució Catalana de Recerca i Estudis Avançats, 08010 Barcelona, Spain \\ *e-mail: romain.quidant@icfo.es
}

The implementation of optical tweezers ${ }^{1}$ at a surface opens a huge potential towards the elaboration of future lab-on-a-chip devices entirely operated with light ${ }^{2}$. The transition from conventional three-dimensional (3D) tweezers to $2 \mathrm{D}$ is made possible by exploiting evanescent fields bound at interfaces ${ }^{3-5}$. In particular, surface plasmons (SP) at metal/dielectric interfaces are expected to be excellent candidates to relax the requirements on incident power and to achieve subwavelength trapping volumes ${ }^{6,7}$. Here, we report on novel 2D SP-based optical tweezers formed by finite gold areas fabricated at a glass surface. We demonstrate that SP enable stable trapping of single dielectric beads under nonfocused illumination with considerably reduced laser intensity compared with conventional optical tweezers. We show that the method can be extended to parallel trapping over any predefined pattern. Finally, we demonstrate how SP tweezers can be designed to selectively trap one type of particles out of a mixture, acting as an efficient optical sieve.

During the past decade, the emergence of 'plasmonics' has given rise to several important breakthroughs in the control, enhancement and confinement of surface optical fields. In particular, the control of surface plasmons (SP) has become increasingly attractive for optical signal processing ${ }^{8}$, surfaceenhanced spectroscopy ${ }^{9-11}$ and sensor technology ${ }^{12}$. Parallel to this research effort, confinement and intensity of SP fields suggest new promising breakthroughs in the topical area of optical manipulation and transport of tiny amounts of matter. In this context, fully integrated optical tweezers able to produce versatile and controllable optical force landscapes are highly desirable for the development of new lab-on-a-chip devices. Such an alternative method replaces single-beam three-dimensional (3D) optical trapping technology, which usually operates with cumbersome bulk optics and relatively high laser intensity, with engineered plasmonic patterns able to work with non-focused illumination and a lower laser intensity threshold.

In a preliminary work, using a photonic force microscope $e^{13}$, we have directly measured the forces induced on single dielectric micro-beads by an SP field generated at the surface of a thin homogeneous gold layer. When working at the SP resonance, the total force magnitude on the probe bead was found to be 40 times stronger than the force measured in the absence of SP excitation. Other measurements have revealed ${ }^{14}$ how organization of large assemblies of colloidal particles into hexagonal patterns can be triggered by SP fields. In this case, thermal convection resulting from the enhanced metal absorption at plasmon resonance also contributes to the self-assembly process.

A flat gold surface illuminated by an asymmetrical non-focused laser beam leads to a homogeneous in-plane optical potential that does not allow localized trapping of single objects to be carried out. Local trapping requires an additional confinement in the surface plane which can be introduced by patterning the metal surface. Whereas the exponential decay of the field intensity away from the surface maintains the object close to the plane surface, the in-plane intensity gradient around the metal structures must be engineered to design a stable trapping well.

Here, we report on the first implementation of 2D SP-based optical tweezers for the manipulation of single micrometre-sized dielectric beads. Our experimental observations are supported by calculations on the basis of the implementation of a 3D Green dyadic function approach.

The optical configuration of the experiment is shown in Fig. 1. A gold pattern, lithographically designed at the surface of a glass sample, is illuminated under total internal reflection by a linearly $p$-polarized laser beam $\left(\lambda_{0}=785 \mathrm{~nm}\right)$ through a hemicylindrical glass prism. The incident beam waist at the glass/water interface is adjusted to about $100 \mu \mathrm{m}$. The power at the entrance of the prism is fixed at $500 \mathrm{~mW}$, corresponding to an intensity $I=5 \times 10^{7} \mathrm{~W} \mathrm{~m}^{-2}$. This intensity is more than one order of magnitude weaker than the minimum intensity required in a conventional 3D optical trap formed by a tightly focused laser beam $\left(1 \mathrm{~mW}\right.$ in $1 \mu \mathrm{m}^{2}$, that is, $10^{9} \mathrm{~W} \mathrm{~m}^{-2}$ ). The gold structures are fabricated using electron-beam lithography combined with a lift-off process. For all geometries considered in this study, the gold thickness is $40 \mathrm{~nm}$. Before carrying out the measurements, a $20-\mu \mathrm{m}$-deep chamber containing a diluted aqueous solution of monodispersed $4.88 \mu \mathrm{m}$ polystyrene spheres (optical index $n=1.59$ ) is prepared on top of each fabricated substrate (concentration $=0.012 \%$ ).

As control experiments, we first considered the case of a homogeneous gold surface illuminated under SP resonance conditions (incident angle $\Phi=68^{\circ}$ ) and, second, a bare glass surface under the same illumination conditions. For a bare glass surface, colloidal particles are attracted towards the surface by the gradient forces and guided by the scattering forces along the incident in-plane $k$-vector ${ }^{3}$. For the illumination conditions considered in this study, the average guiding velocity is about $6 \mu \mathrm{m} \mathrm{min}^{-1}$. Conversely, for the chamber depth and the illumination conditions we use, the dynamics of the colloids in 
a

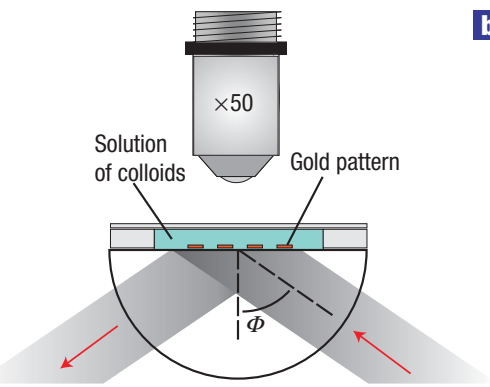

b

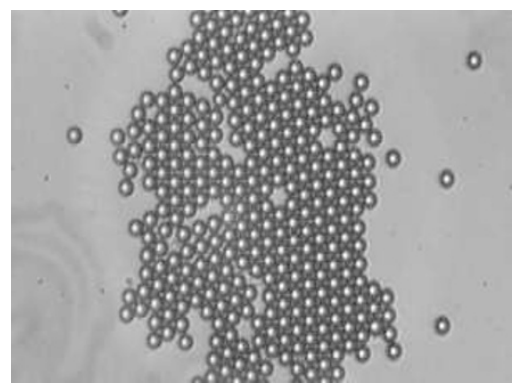

G

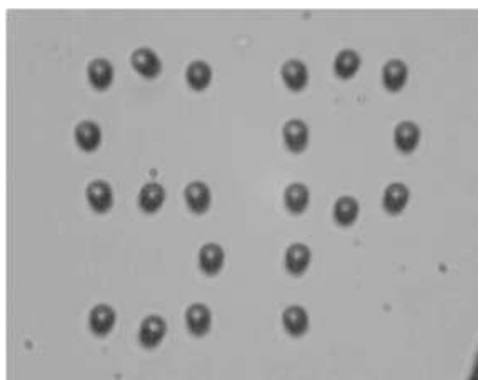

Figure 1 SP-induced organization at a metal surface. a, Schematic diagram of the optical configuration. b,c, Arrangement of polystyrene micro-beads at a homogeneous (b) and a patterned (c) gold/water interface after laser illumination at the SP resonance.

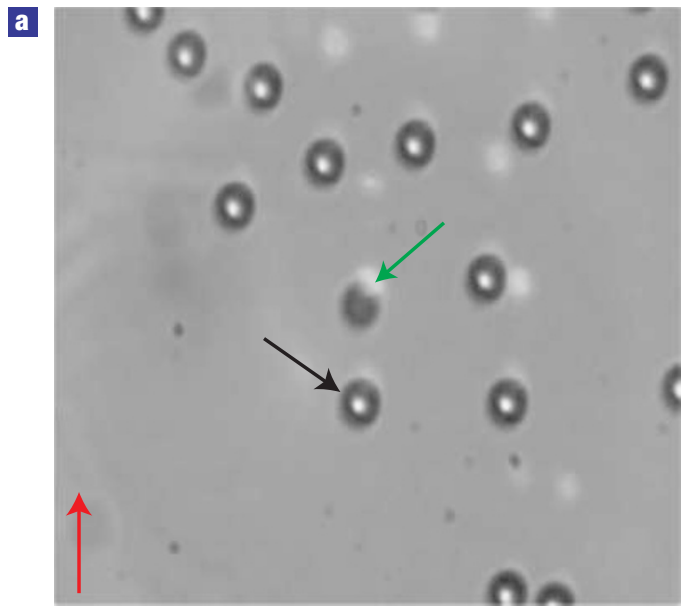

G

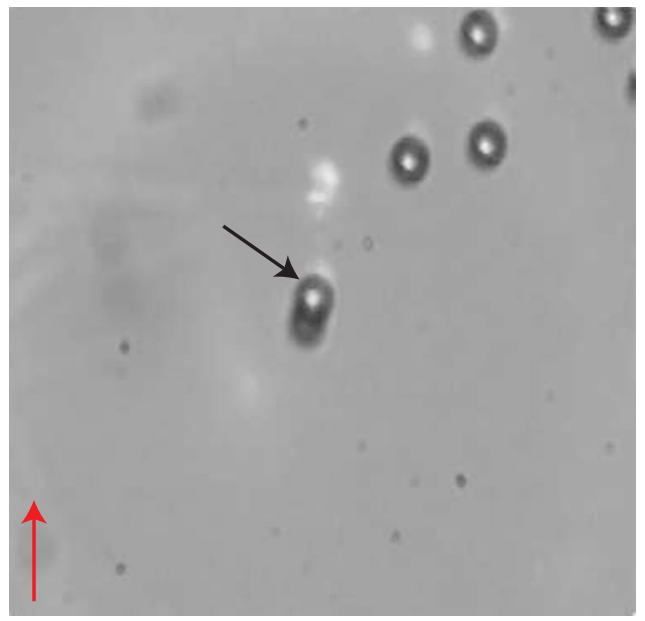

b

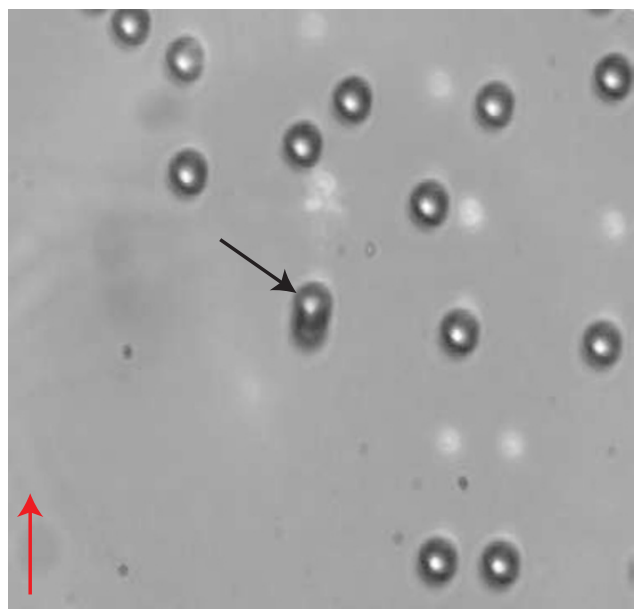

d

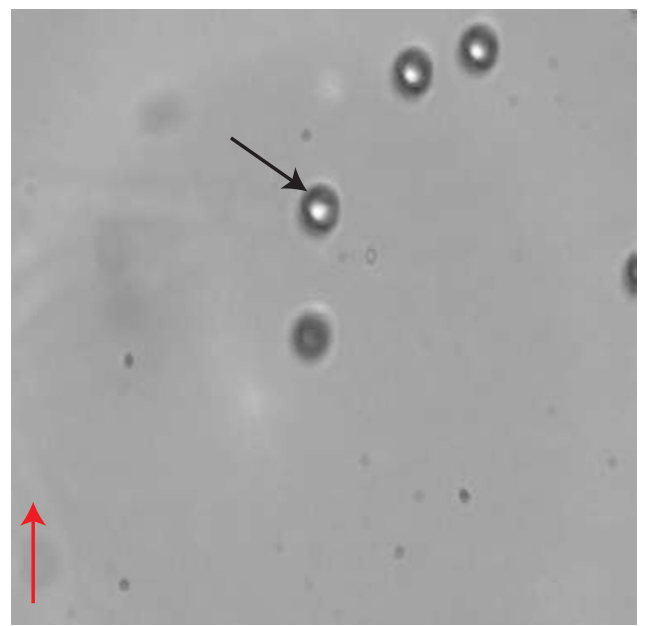

Figure 2 Trapping at a single micro-SP trap. a-d, A sequence of images recorded above an isolated 4.8- $\mu$ m-diameter gold disc (marked by the green arrow in a). The red arrow points along the incident in-plane $k$-vector. For $\mathbf{d}$, the polarization state is switched from $p$ to $s$.

the presence of the gold layer is governed by a combination of thermal and optical contributions ${ }^{14}$. Thermal convection effects tend to gather the particles towards the centre of the illumination area, whereas the gradient optical force arising from the vertical exponential decay of the SP field tends to maintain the beads in the surface plane. Figure $1 \mathrm{~b}$ shows a typical compact hexagonal area formed after approximately $10 \mathrm{~min}$ under laser illumination.
By patterning the metal film, we can create local SP excitations in predefined positions. In this way, strong gradients of the optical near-field intensity are expected when passing from the metal to the glass. The resulting field pattern should give rise to an optical potential landscape able to influence the dynamic of the colloid flow. This approach offers several advantages over other evanescent methods such as the one using a total-internal-reflection objective 


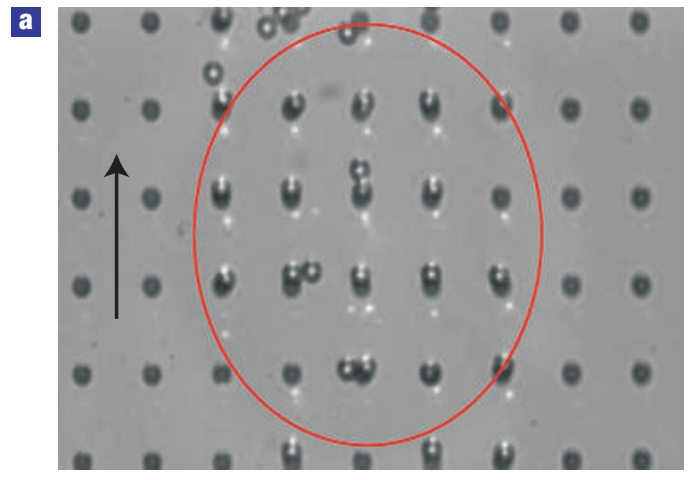

G

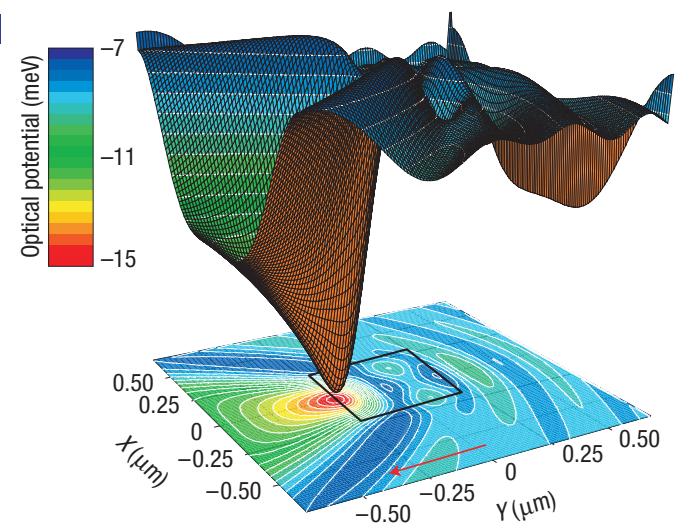

b

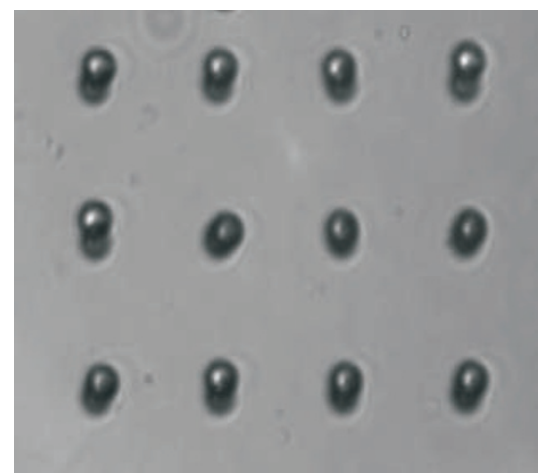

d

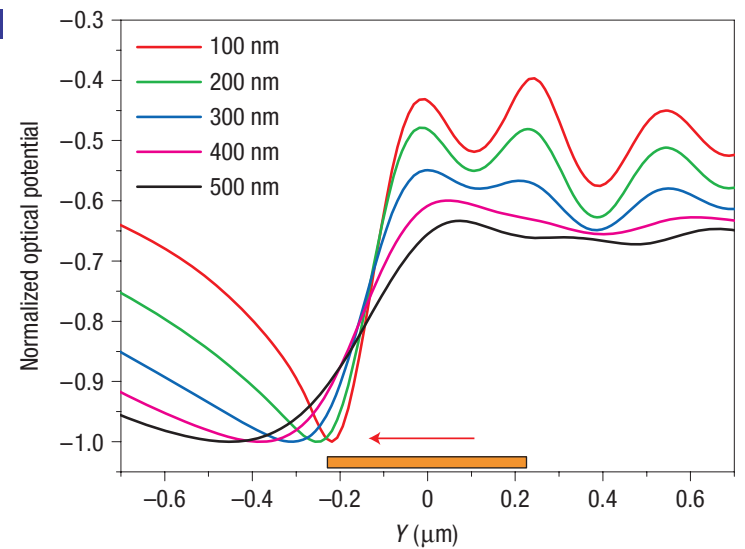

Figure 3 Parallel trapping and trapping mechanism. a, Parallel trapping of $4.88 \mu \mathrm{m}$ polystyrene colloids over a periodic pattern formed by $4.8-\mu \mathrm{m}$-diameter gold discs after $15 \mathrm{~min}$ illumination. The ellipse delimitates the illumination area and the arrow points along the incident in-plane $k$-vector. $\mathbf{b}$, Image recorded during the same experiment after $30 \mathrm{~min}$ on a smaller area. The incident laser is filtered for better visibility. c, 3D view of an optical binding energy trap computed for a $200 \mathrm{~nm}$ polystyrene bead near a $0.45-\mu \mathrm{m}$-side square gold pad. d, Cross-sections of the binding energy trap along the $Y$ direction computed for different bead sizes.
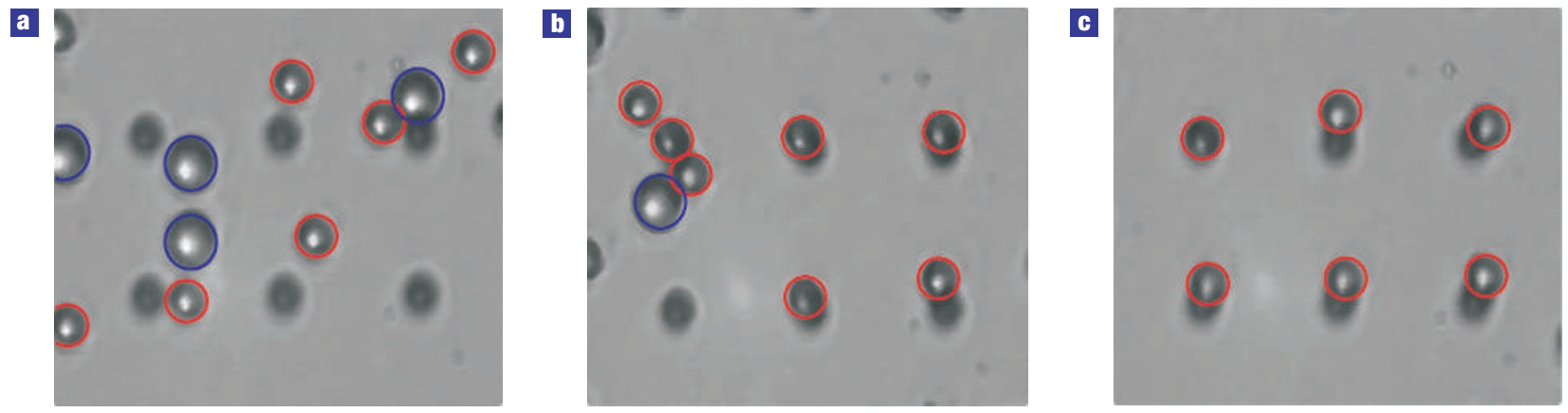

Figure 4 Selectivity of the SP traps to the colloids size. The 3.5- $\mu \mathrm{m}$-diameter trap area is exposed to a mixture of $3.55 \mu \mathrm{m}$ (in red) and $4.88 \mu \mathrm{m}$ (in blue) polystyrene colloids. The three successive images are each separated by $7 \mathrm{~min}$.

lens ${ }^{15}$. In particular, it does not require laser focusing through a bulk objective, enables multiple trapping from a single beam and benefits from the local field enhancement of SP at the metal surface.

The first pattern we consider is formed by a single gold disc of micrometric diameter $(4.8 \mu \mathrm{m})$. Figure 2 shows a sequence of successive images recorded above this simple object restituting the dynamical behaviour of $4.88 \mu \mathrm{m}$ polystyrene beads. In the present situation, where the area occupied by the metal is weak, multidirectional motion of the beads owing to large thermal convection phenomena is significantly reduced to become localized around the gold disc. It can be seen, in Fig. $2 a-c$, that one of the beads (marked by the black arrow), initially guided along the glass surface by the scattering force, is suddenly trapped when approaching the gold pad. Despite its brownian motion, the 
bead stays confined to a portion of the pad area as long as the illumination is maintained. Similar behaviours have been observed for polystyrene beads with sizes ranging from $3 \mu \mathrm{m}$ to $5.5 \mu \mathrm{m}$. Note that owing to the weak optical index difference between polystyrene and water, the forces on the beads are not expected to be influenced by whispering-gallery resonances ${ }^{4}$. To verify that this trapping mechanism is triggered by the SP and to rule out any other chemical or electrostatic interactions, the polarization of the incident laser was switched, in Fig. 2 d, from $p$ to $s$ mode where no SP resonance is expected. In this polarization, the significant decrease of the near-field intensity above the gold disc makes the scattering forces and the brownian fluctuations overcome the restoring forces. Consequently, after about $1 \mathrm{~min}$ we observe that the bead frees itself from the gold pad. We also observe a similar behaviour under $p$-polarization when changing the incident angle to a value that does not match the SP resonance angle.

To demonstrate the flexibility of SP tweezers, the experiment is now extended to an array of gold pads. Figure $3 \mathrm{a}$ shows the observation made, after $15 \mathrm{~min}$, on a square pattern of $4.8-\mu \mathrm{m}$ diameter gold discs separated by $25 \mu \mathrm{m}$. It can be seen that most of the illuminated pads ( 21 out of 26 or about $80 \%$ ) are occupied by a single bead. The presence of some empty sites can be attributed to the inhomogeneous distribution of the particle flow. We also note that there are no trapped beads outside the illumination area.

From Fig. 3b, it can be seen that the trapped beads stabilize in a forward position with respect to the gold pads centre. To gain further insight into the trapping mechanism, extensive simulations have been carried out using the Green dyadic method ${ }^{16}$ combined with an optical binding energy computation above the gold pad. This volume integration method is particularly well suited to visualize the shape evolution of the trapping well as a function of the different experimental parameters. To maintain the calculation time to a reasonable level, the simulation considers a $0.45-\mu \mathrm{m}$-side square gold pad. Figure $3 \mathrm{c}$ shows a map of the optical potential computed for a $200 \mathrm{~nm}$ polystyrene bead located $20 \mathrm{~nm}$ above the gold pad under the same illumination conditions as in the experiment. It shows a deep and confined potential well located in a forward position, corroborating very well our experimental observations. The associated near-field intensity map (not shown) reveals that this shift mainly arises from the illumination asymmetry leading to a significant forward-scattering from the gold pad. Note that the unidirectional character of the scattering force (which does not contribute to the potential shaping) further contributes to the shifted equilibrium position of the bead. Although this purely electromagnetic mechanism satisfactorily explains our observations, other additional effects could be evoked. In particular, the local plasmon heating process could reinforce the optical binding trap by creating a convection stream around the metallic pad. To describe this mechanism, we consider the total heat, $Q$, dissipated (per time unit) by the gold discs. This dissipated power is known to be proportional to the imaginary part of the metal dielectric function, $\epsilon^{\prime \prime}\left(\omega_{0}\right)$ (where $\omega_{0}=2 \pi c / \lambda_{0}$, where $\lambda_{0}$ is the incident wavelength in vacuum).

$$
Q=\frac{\omega_{0}}{8 \pi} \int_{v} \epsilon^{\prime \prime}\left(\omega_{0}\right)\left|\mathcal{E}\left(\omega_{0}, \mathbf{r}\right)\right|^{2} \mathrm{~d} \mathbf{r}
$$

where $\varepsilon\left(\omega_{0}, \mathbf{r}\right)$ is the local self-consistent electric field inside the metal and where the integral runs over the volume of the gold particle. At the SP resonance, $\mathscr{E}\left(\omega_{0}, \mathbf{r}\right)$ takes important values at the surface of the gold disc. Consequently, the plasmonic metal will release significant amounts of energy to the liquid, particularly in the regions where we usually observe an enhancement of the nearfield intensity. The very localized convection streams that result from this off-equilibrium process are enhanced when reaching the SP resonance of the gold structure. They may play a significant role in the trapping process observed in Fig. 2b. Whereas the SP forces magnitude increases with the particle diameter, the confinement of the trap decreases and get shallower (see Fig. 3d). This specificity can be exploited to achieve a trapping selectivity for different particle sizes. For a given gold pad, there will be a particle diameter for which the scattering force (also increasing with particle size) will exceed the restoring SP forces. In practice, because particles of the same size as the pad diameter are still efficiently trapped, this is expected to occur for significantly bigger particles.

To verify this concept, we have carried out an additional experiment where the solution contains an equal proportion of two polystyrene bead sizes $(4.88 \mu \mathrm{m}$ and $3.55 \mu \mathrm{m})$. The gold pads with a diameter of $3.50 \mu \mathrm{m}$ have been designed to achieve a preferential trapping of the smallest beads. Figure 4 shows a set of three successive images recorded with a $7 \mathrm{~min}$ interval time. Although both particle sizes have a similar probability to pass the trapping area, after $21 \mathrm{~min}$, only the $3.55 \mu \mathrm{m}$ particles are trapped.

SP tweezers open new perspectives to transport, trap and sort with light small objects at the surface of a chip. Future work will address their implementation to volumes of matter smaller than the incident wavelength and living biological entities.

\section{Received 6 January 2007; accepted 23 April 2007; published 21 May 2007.}

\section{References \\ 1. Grier, D. G. A revolution in optical manipulation. Nature 424, 810-816 (2003). \\ 2. Gluckstad, J. Microfluidics: Sorting particles with light. Nature Mater. 3, 9-10 (2004). \\ 3. Kawata, S. \& Sugiura, T. Movement of micrometer-sized particles in the evanescent field of a laser beam. Opt. Lett. 17, 772-774 (1992). \\ 4. Kawata, S. \& Tani, T. Optically driven Mie particles in an evanescent field along a channeled waveguide. Opt. Lett. 21, 1768-1770 (1996). \\ 5. Cizmár, T. et al. Optical sorting and detection of submicrometer objects in a motional standing wave. Phys. Rev. B 74, 035105 (2006) \\ 6. Chaumet, P. C., Rahmani, A. \& Nieto-Vesperinas, M. Optical trapping and manipulation of nano-objects with an apertureless probe. Phys. Rev. Lett. 88, 123601 (2002). \\ 7. Xu, H. \& Kall, M. Surface-plasmon-enhanced optical forces in silver nanoaggregates. Phys. Rev. Lett. 89, 246802 (2002). \\ 8. Barnes, W. L., Dereux, A. \& Ebbesen, T. W. Surface plasmon subwavelength optics. Nature 424 $824-830$ (2003). \\ 9. Nie, S. \& Emory, S. T. Probing single molecules and single nanoparticles by surface-enhanced Raman scattering. Science 275, 1102-1106 (1997). \\ 10. Jordan, P. et al. Surface enhanced resonance Raman scattering in optical tweezers using co-axial second harmonic generation. Opt. Express 13, 4148-4153 (2005). \\ 11. Svedberg, F., Li, Z., Xu, H. \& Kall, M. Creating hot nanoparticle pairs for surface-enhanced Raman spectroscopy through optical manipulation. Nano Lett. 6, 2639-2641 (2006). \\ 12. McFarland, A. D. \& Van Duyne, P. Single silver nanoparticles a real-time optical sensing sensors with zeptomole sensitivity. Nano Lett. 3, 1057-1062 (2003). \\ 13. Volpe, G., Quidant, R., Badenes, G. \& Petrov, D. Surface plasmon radiation forces. Phys. Rev. Lett. 96, 238101 (2006). \\ 14. Garcés-Chávez, V. et al. Extended organization of colloidal microparticles by surface plasmon polariton excitation. Phys. Rev. B 73, 085417 (2006). \\ 15. Gu, M., Haumonte, J. B., Micheau, Y., Chon, J. W. M. \& Gan, X. Laser trapping and manipulation under focused evanescent wave illumination. Appl. Phys. Lett. 84, 4236-4238 (2004). \\ 16. Girard, C., Dereux, A. \& Martin, O. J. F. Theoretical analysis of light-inductive forces in scanning probe microscopy. Phys. Rev. B 49, 13872-13881 (1994).}

\section{Acknowledgements}

This research was carried out with financial support from the European Commission through grant ATOM3D FP6-508952 and the Network of Excellence 'Plasmon Nano-devices' (PND)-FP6-507 879. Correspondence and requests for materials should be addressed to R.Q.

\section{Author contributions}

M.R. designed the samples and carried out the experiments. A.Z. and C.G. carried out the numerical simulations. R.Q. planned the project, designed the experiment and wrote the paper with support from C.G. All of the authors participated in the analysis of the results.

Competing financial interests

The authors declare no competing financial interests.

Reprints and permission information is available online at http://npg.nature.com/reprintsandpermissions/ 